\title{
Urgences
}

\section{Jacques Beaudry, La philosophie \& le Québec : des noms et des notes. I, Sherbrooke, Ex-Libris, 1989, 205 p.}

\section{Jean-Claude Simard}

Numéro 30, décembre 1990

L'autre du texte

URI : https://id.erudit.org/iderudit/025630ar

DOI : https://doi.org/10.7202/025630ar

Aller au sommaire du numéro

\section{Éditeur(s)}

Urgences

\section{ISSN}

0226-9554 (imprimé)

1927-3924 (numérique)

Découvrir la revue

Citer ce compte rendu

Simard, J.-C. (1990). Compte rendu de [Jacques Beaudry, La philosophie \& le Québec : des noms et des notes. I, Sherbrooke, Ex-Libris, 1989, 205 p.] Urgences, (30), 111-114. https://doi.org/10.7202/025630ar d'utilisation que vous pouvez consulter en ligne.

https://apropos.erudit.org/fr/usagers/politique-dutilisation/ 


\section{deliveline}

\section{Jacques Beaudry, La philosophie \& le Qué- bec: des noms et des notes. 1, (Sherbrooke, Ex Libris, 1989, 205 p.)}

La philosophie, si elle est soucl de la condition humaine, est peut-etre aussi recherche d'un pays.

(F. Dumont, cité p. 54)

L'histoire de la philosophie québecoise est un domaine encore en friche. En particulier en ce qui a trait à la période contemporaine. Les longues controverses des années 1970 se sont surtout limitées à établir la légitimité du champ de recherche. C'est alors qu'on a pu en esquisser les contours sous le mode de l'analyse institutionnelle (Lamonde), ou encore l'éclairer par la bande. Ces lueurs indirectes sont venues de la problématique des idéologies d'abord (les travaux pionniers dirigés par Dumont, ceux de Bernard, sans oublier, bien sûr, le ćlèbre ouvrage de Monière), mais aussi de réflexions sur l'essai philosophique (Houde, MarcilLacoste) ou de coups de sonde résolument non conformistes (Chabot et Vidricaire). On en est actuellement à la période des travaux plus pointus. C'est dans œ contexte précis qu'il faut, à mon avis, situer le présent travail.

Depuis plusieurs années en effet, Beaudry s'attache à retracer des * itinéraires intellectuels * - c'est le sous-titre de l'un de ses ouvrages les plus significatifs
- et a fournir aux chercheurs ou aux simples curieux des instruments d'exploration. Dans la première catégorie figurent déjà des essais sur Lavigne (Autour de Jacques Lavigne, philosophe, 1985) et Houde (Roland Houde, un philosophe et sa circonstance, 1986). Pour ce qui est de la seconde catégorie, on peut signaler a titre d'exemple Philosophie et périodiques québécois (1983).

D'une certaine manière, La philosophie \& le Québec n'est pas, comme tel, une ouvre d'histoire de la philosophie, du moins au sens habituel du terme. Décrivant son type d'approche assez particulier, l'auteur évoquait dans un ouvrage antérieur les * fragments d'une histoire parabiographique toujours a faire * (Autour de Jacques Lavigne, philosophe, p. 7). Il reprend ici cette voie d'accès puisqu'il préconise une * transformation radicale de la perspective en histoire de la philosophie au Québec* (p. 9). Une telle transformation délaisserait *l'historiographie habituelle * pour faire fond sur la biographie intellectuelle, ce que l'auteur appelle pour sa part la * parabiographie *, c'est-a-dire le tracé « d'itinéraires intellectuels copieux, diffus et drus, bourrés (sic) de documents, de notes et de références *. Et c'est bien ainsi que se présente cet ouvrage, alignant alphabétiquement de brèves notices qui, sans présenter un format véritablement uniforme, retracent le plus souvent le parcours d'un auteur, son chemin de pensée, pour parler comme Heidegger. On y met surtout en relief le rapport a la philosophie - d'ailleurs enten- 
due en un sens aussi peu technique qu'académique -, non sans offrir au passage de généreux extraits assortis de copieuses orientations bibliographiques. Ainsi, comme ne manque pas de le signaler l'auteur luimême, aucune de ces notices n'a de prétention à l'exhaustivité, et aucune ne répète vraiment, malgré d'inévitables recoupements, ce qu'on pourra de toute façon trouver dans les repertoires habituels, qu'il s'agisse du Dictionnaire pratique des auteurs québécois ou du Dictionnaire des cuvres littéraires du Québec, pour ne nommer que les deux plus connus.

Pour ce qui est des auteurs retenus, Beaudry reconnait volontiers l'arbitraire de ses choix. Malgré quoi, on peut déceler quelques constantes et reconstituer certains des critères qui semblent avoir opéré. Ainsi, l'on note immédiatement qu'hormis Edmond de Nevers, les 81 auteurs mentionnés ont tous travaille au XX' siecle. Et $\propto$ qui retient encore aussitôt l'attention, c'est le lien de chacun à la * question du Québec* (Rioux). Ainsi s'éclaire le sens du titre: La philosophie \& le Québec, qu'il faut entendre non pas comme $L a$ philosophie au Québec, mais bien plutôt comme La philosophie et le fait québécois. C'est pourquoi sans doute sont traités quasi exclusivement, même chez les contemporains, les philosophes qui sont proches des questions sociopolitiques ou de la littérature, plutôt que, par exemple, ceux qui se réclament plus spontanément des philosophies du langage ou des sciences, peutêtre les deux courants dominants sur la scène québécoise actuelle. À première vue, la chose semble evidemment de nature a limiter la portée de l'ouvrage. Mais ne prejugeons pas trop: comme il ne s'agit pour l'instant que du premier tome, peut-être finira-t-on par y retrouver la plupart des philosophes québécois majeurs, quelle que soit par ailleurs leur allégeance.

Tel qu'il se présente en tout cas, l'ensemble a, malgré sa peu orthodoxe facture (ou peut-être bien à cause d'elle), l'attrait des rencontres imprévues, le charme des vagabondages livresques. Beaudry n'a pas craint l'aventure hors des sentiers battus et il faut lui en donner crédit, même si parfois, me semble-t-il - et c'est le seul véritable reproche que pourrait encourir l'ouvrage - , il n'a pas toujours su faire le départ entre le parti pris et le franc subjectivisme, celui-la inopportun, celui-ci inévitable sans doute, souhaitable même en pareille matière. Sans qu'on sache en effet très bien pourquoi, d'illustres inconnus surgissent ça et la qui, leur notice l'atteste, avaient pleinement mérité leur anonymat.

Ce qui fait malgre tout l'intérêt manifeste de l'ouvrage, c'est la multiplication des approches qu'il autorise: interprétations plurielles marquées au coin de carrefours intertextuels et de leurs résonances historiques. Cette pluralité est d'ailleurs largement facilitée par un index des noms de même qu'un index analytique très complets. Ajoutons encore à cela l'étonnante diversité des sources compulsées: livres, revues et périodiques, bien sûr, mais aussi mémoires, thèses, travaux d'etudiants, répertoires,catalogues, gazettes, quotidiens, inédits issus de conférences, de colloques, etc. Pour son plaisir (et le nôtre), l'auteur a énormément lu et beaucoup dépouillé. L'explora- 
teur du dimanche qui sommeille en chacun de nous aura loisir de découvrir, au fil des pages, au détour d'un nom, ces petites surprises agréables, ces aperçus soudains, ces faits étonnants et en apparence anodins dont le complexe entrelacs finit par suggérer le sens d'un parcours, le parfum d'une cuvre, voire le ton d'une époque. À titre d'exemples: le mémoire de licence en philoso. phie de Raoul Duguay, Aquin et son film sur le sport, ô raison de la raison, la pièce de théatre de Chabot et Vidricaire (en collabo. ration avec Barette et Pinard), la formation essentiellement philosophique de Michèle Lalonde, et combien d'autres encore !

Pourtant, Beaudry n'approfondit lui-même aucun sujet précis. Il se contente d'entr'ouvrir des portes. Ainsi, l'ensemble abonde en références évocatrices, en renvois bibliographiques, en orientations de lecture, en avenues de recherche, en parcours critiques, en problematiques quasi vierges. Invitation a une approche libidinale qui, partant de là, saurait entrecroiser les séries signifiantes au gré des appels, des rejets, des contrepoints, des ruptures, des fractures même: bribes flottantes de sens, constellations théoriques, tranches de vie... Les œuvres et les hommes en somme. On l'aura à présent compris: La philosophie \& le Québec multiplie les pistes exploratoires de même que les auteurs aptes a les illustrer. $Q u$ 'il suffise ici d'indiquer au passage quelques repères possibles, avec leurs points d'ancrage respectifs: littérature et philosophie ou les limites disciplinaires et l'artificialité des genres (Aquin, Bellow, Brault, Kérouac, Thériault), poésie et philosophie ou l'irruption de l'indicible dans le domaine $d u$ pensable (Chamberland, Dumont, Haeck, Miron, Saint-Denys Garneau), philosophie et essai ou l'impossible et nécessaire mariage (Borduas, Hertel, Simard, Vadeboncour), séjours québecois et fécondation interculturelle (Camus, Marcel, Sartre), québécitude enfin et histoire de la philosophie (Cauchy, Chabot et Vidricaire, Dumont, Hébert, Houde, Lamonde, Marcil-Lacoste).

Sans prétention indue, Beaudry offre donc un instrument de travail dans un domaine ou ils se comptent sur les doigts d'une seule main. Malgré ses limites, La philosophie \& le Québec intéressera les philosophes curieux de leur histoire, bien sûr, mais aussi tous ceux et celles qui, plus généralement, se piquent de pensée québécoise. Car il constitue en définitive un hommage aux devanciers, à ceux et celles qui, avant nous, ont peiné pour aménager ces rares éclaircies où nous frayons encore tant bien que mal.

Voulant stigmatiser ceux et celles qui croient la philosophie depuis toujours absente des forces agissantes de notre scène nationale, et donc aussi du champ effectif de notre histoire, Marcil-Lacoste parlait, il y a quelques annees à peine, d'un $* a$ priori de l'improductivité *. Sa sensibilité à la chose historique aidant, comme aussi son sens aigu de l'archive, Beaudry nous rappelle une fois encore que, a l'instar des arts et de la littérature, secrère complice parfois, la philosophie a ici une tradition. Qui demande réappropriation dans une intimite distanciée, qui exige remémoration dans une oublieuse culture. Car, pour être encore et toujours a l'état de chantier, comme l'avait bien vu Dumont, une telle entreprise 
114

n'en demeure pas moins essentielle autant qu'urgente. Et elle ne saurait se mettre en branle sans que soient rassemblés de nouveaux matériaux, sans que soient forgés de nouveaux outils. Cet ouvrage en constitue un parmi d'autres.

Vivement, le tome 2!

Jean-Claude Simard 